\title{
Manufacture of Clinical-Grade Human Clonal Mesenchymal Stem Cell Products from Single Colony Forming Unit-Derived Colonies Based on the Subfractionation Culturing Method
}

\author{
TacGhee Yi, PhD, ${ }^{1-3}$ Si-na Kim, MS, ${ }^{4}$ Hyun-Joo Lee, MS, ${ }^{4}$ Junghee Kim, BS, ${ }^{4}$ Yun-Kyoung Cho, MS, ${ }^{3}$ \\ Dong-Hee Shin, MS, ${ }^{1,2}$ Sun-Ji Tak, AS, ${ }^{1}$ Sun-Hwa Moon, AS, ${ }^{3} \mathrm{Ji}-E u n$ Kang, BS, ${ }^{3}$ In-Mi Ji, MS,, \\ Huyn-Ja Lim, MS, ${ }^{3}$ Dong-Soon Lee, MD, PhD, ${ }^{5}$ Myung-Shin Jeon, $\mathrm{PhD}$, and Sun U. Song, PhD ${ }^{1,3}$
}

Stem cell products derived from mesenchymal stem cells (MSCs) have been widely used in clinical trials, and a few products have been already commercialized. However, the therapeutic effects of clinical-grade MSCs are still controversial owing to mixed results from recent clinical trials. A potential solution to overcome this hurdle may be to use clonal stem cells as the starting cell material to increase the homogeneity of the final stem cell products. We have previously developed an alternative isolation and culture protocol for establishing a population of clonal MSCs (cMSCs) from single colony forming unit (CFU)-derived colonies. In this study, we established a good manufacturing practice (GMP)-compatible procedure for the clinical-grade production of human bone marrowderived cMSCs based on the subfractionation culturing method. We optimized the culture procedures to expand and obtain a clonal population of final MSC products from single CFU-derived colonies in a GMP facility. The characterization results of the final cMSC products met our preset criteria. Animal toxicity tests were performed in a good laboratory practice facility, and showed no toxicity or tumor formation in vivo. These tests include single injection toxicity, multiple injection toxicity, biodistribution analysis, and tumorigenicity tests in vivo. No chromosomal abnormalities were detected by in situ karyotyping using oligo-fluorescence in situ hydridization (oligo-FISH), providing evidence of genetic stability of the clinical-grade cMSC products. The manufacture and quality control results indicated that our GMP methodology could produce sufficient clonal population of MSC products from a small amount of bone marrow aspirate to treat a number of patients.

\section{Introduction}

$\mathbf{S}$ TEM CELL THERAPY has emerged as a promising treatment for a variety of incurable diseases. ${ }^{1}$ Stem cells have been shown to be therapeutically beneficial in a number of experimental diseases. Clinical-grade production of stem cells is a prerequisite for a broad range of clinical applications. However, being quite different from laboratory stem cells for basic and translational studies, clinical-grade stem cells suitable for patients cannot be simply produced by using a laboratory methodology. Good manufacturing practice (GMP), a quality assurance system in the pharmaceutical industry, should be employed to produce clinical-grade stem cell products with defined quality. ${ }^{2,3}$ Stem cell production in a GMP environment cannot be achieved by the simple transfer of laboratory-scale stem cell isolation and culture procedures. The final stem cell products must fulfill the GMP standards, and the establishment of a validated standard operation procedure (SOP) for the whole manufacturing and quality control processes is essential. ${ }^{2}$ In terms of commercialization of clinical research, mesenchymal stem cells (MSCs) appear to be a step ahead of other stem cell types as one of the most valuable model of stem cell products. ${ }^{4}$ In fact, some stem cell products made of MSCs have been already commercially

\footnotetext{
${ }^{1}$ Translational Research Center, Inha University School of Medicine, Incheon, Republic of Korea.

${ }^{2}$ Inha Research Institute for Medical Science, Inha University School of Medicine, Incheon, Republic of Korea.

${ }^{3}$ SCM Lifescience Co., Ltd., Incheon, Republic of Korea.

${ }^{4}$ Drug Development Program, Department of Biomedical Science, Inha University School of Medicine, Incheon, Republic of Korea.

${ }^{5}$ Department of Pathology, Seoul National University School of Medicine, Seoul, Republic of Korea.
}

(c) T. Yi et al. 2015; Published by Mary Ann Liebert, Inc. This Open Access article is distributed under the terms of the Creative Commons Attribution Noncommercial License (http://creativecommons.org/licenses/by-nc/4.0/) which permits any noncommercial use, distribution, and reproduction in any medium, provided the original author(s) and the source are credited. 
approved, but their availability is restricted to Korea, Canada, and New Zealand. Nonetheless, the therapeutic effects of clinical-grade MSCs are still controversial owing to mixed results from recent clinical trials. ${ }^{4-6}$ Clinical trials performed over the last decade have validated the safety of clinicalgrade MSCs, ${ }^{7,8}$ but the mixed results have showed both optimistic and pessimistic prospects about the efficacy of their products. One of the possible reasons for the mixed results may be the heterogeneity of the final stem cell products. There is a consensus that not all MSCs are identical. ${ }^{5}$ It is unclear how to generate consistently effective MSCs by using the current stem cell culture techniques. Moreover, although MSCs have shown potent beneficial effects in experimentally controlled translational studies, it is unclear how MSCs work in the patients' immune system. Therefore, there exists an incomplete understanding of the effectiveness of MSCs and the lack of the technologies to accurately control them. Furthermore, others and we have reported the existence of different MSC populations with nonidentical characteristics even in the same batch of MSCs, indicating the cellular heterogeneity of MSCs and the technical limitations of the current stem cell production methodology. ${ }^{9-11}$ A potential solution to overcome this hurdle may be to use clonal stem cells as the starting cell material to maximize the homogeneity of the final stem cell products. We have previously developed an alternative culture protocol based on clonal selection, named subfractionation culturing method (SCM), to produce a population of clonal MSCs (cMSCs) from a single colony forming unit (CFU)-derived colonies, which is different from the widely used conventional isolation method. ${ }^{9,12}$ Our method produces a population of cMSCs, while the conventional method yields a heterogeneous pool of MSCs from the source materials. We have also reported the beneficial effects of cMSCs in a variety of animal disease models including acute pancreatitis, graft-versus-host disease (GVHD), salivary hypofunction, and vocal-fold scarring, thus providing evidence of the usefulness of cMSCs. ${ }^{13-16}$ In this study, we established a GMP-compatible procedure for the clinical-grade production of human bone marrow-derived cMSCs based on the SCM. We have optimized the manufacturing procedures to expand and obtain cMSCs sufficient for the final products from single CFU-derived colonies in a GMP facility. In addition, we have evaluated the safety of the final cMSC products in toxicological studies to ensure the safety of the cells.

\section{Materials and Methods}

\section{Isolation and culture of cMSCs}

Bone marrow aspirates were obtained from the iliac crest of three healthy donors after written informed consent (approved by Inha University Hospital Institutional Review Board; IRB number 10-51). Isolation of cMSCs was performed as described previously. ${ }^{9}$ In brief, small human bone marrow aspirate was mixed with $15 \mathrm{~mL}$ of isolation medium: Dulbecco modified Eagle Medium containing low glucose (Gibco-BRL, Life Technologies, Gaithersburg, MD), and with $20 \%$ fetal bovine serum (FBS; Gibco-BRL) and $1 \%$ penicillin/streptomycin (Gibco-BRL), and then incubated in a 100-mm culture dish. As shown in Figure 1A, after incubation for $2 \mathrm{~h}$ at $37^{\circ} \mathrm{C}$ with $5 \% \mathrm{CO}_{2}$, only the cell culture supernatant was transferred to a new 100-mm dish.
After the second 2-h incubation, the supernatant was again transferred to a new dish (D1) and incubated for additional $2 \mathrm{~h}$. The supernatant was transferred to another new dish (D2) and incubated for a day, and then transferred to another new dish (D3) and incubated for a day. This process was repeated two more times with 1- and 2-day incubations (D4 and D5, respectively). In the first three transfers after a short period of incubation, we wanted to let high-density cells, such as white and red blood cells, settle to the bottom of the dish, and the progressively longer intervals between the supernatant transfers would allow the less dense and/or adhesive cells in the supernatant to settle to the bottom of the dish. We hypothesized that this method might allow cells with different densities and/or adherences to be further fractionated. Only well-separated single colonies with a diameter of about $4 \mathrm{~mm}$, ranging from $\sim 4 \times 10^{3}$ to $1 \times 10^{4}$ cells in a single colony, in D2, D3, D4, or D5 dishes were selected and transferred to six-well plates and then to larger culture flasks, where they kept expanding. After 10-14 days in the $100-\mathrm{mm}$ dishes, the single colonies were detached and isolated using a 1 - to 2 -min treatment with $0.05 \%$ trypsin/ EDTA (Gibco-BRL) treatment in cloning cylinders (). Once the cells reached 70-80\% confluence, they were recovered with trypsin/EDTA and replated for further expansion. For cryopreservation, cells harvested were thoroughly washed, filled with cryopreservation medium, which is composed of $10 \%$ pharmaceutical-grade dimethyl sulfoxide (OriGen Biomedical, Korea) and 90\% FBS, and then cryopreserved in a liquid nitrogen tank until thawing.

Due to space limitations, the other parts of materials and methods including cMSC characterization, toxicity tests, and sterility tests are described in Supplementary Materials and Methods (Supplementary Data are available online at www.liebertpub.com/tec). For details refer to Supplementary Materials and Methods.

\section{Results \\ Establishment of a GMP-compatible SOP for the manufacture and quality control of $\mathrm{CMSC}$ production based on SCM}

SCM is capable of producing MSC clones from small aspirates of bone marrow as reported previously. ${ }^{9,17}$ The key issue was whether our laboratory-scale SCM was applicable to GMP-compatible manufacturing procedures to produce sufficient number of cell products for patients in a reproducible manner. We optimized the laboratory-scale SCM protocol to be suitable for large-scale expansion in a GMP facility (Fig. 1A). For MSC isolation from bone marrow aspirates, the basic process was not different from the original protocol. Briefly, the bone marrow aspirates from the ileac crest of a healthy donor were mixed gently and thoroughly in the isolation medium. Repeated transfers of the supernatants containing floating bone marrow cells after the heavier cells settled down (taking up to 5 days) were performed in a 100-mm culture dish. Colonies with high CFU activities were selectively isolated by trypsinization in a cloning cylinder, and they were allowed to grow in a sixwell plate. This step was determined as passage 1. After the growing cells reached about $70 \%$ confluence, the cells were detached and plated to grow in a $75-\mathrm{cm}^{2}$ culture flask (passage 2). From this step, the culture time for a passage 

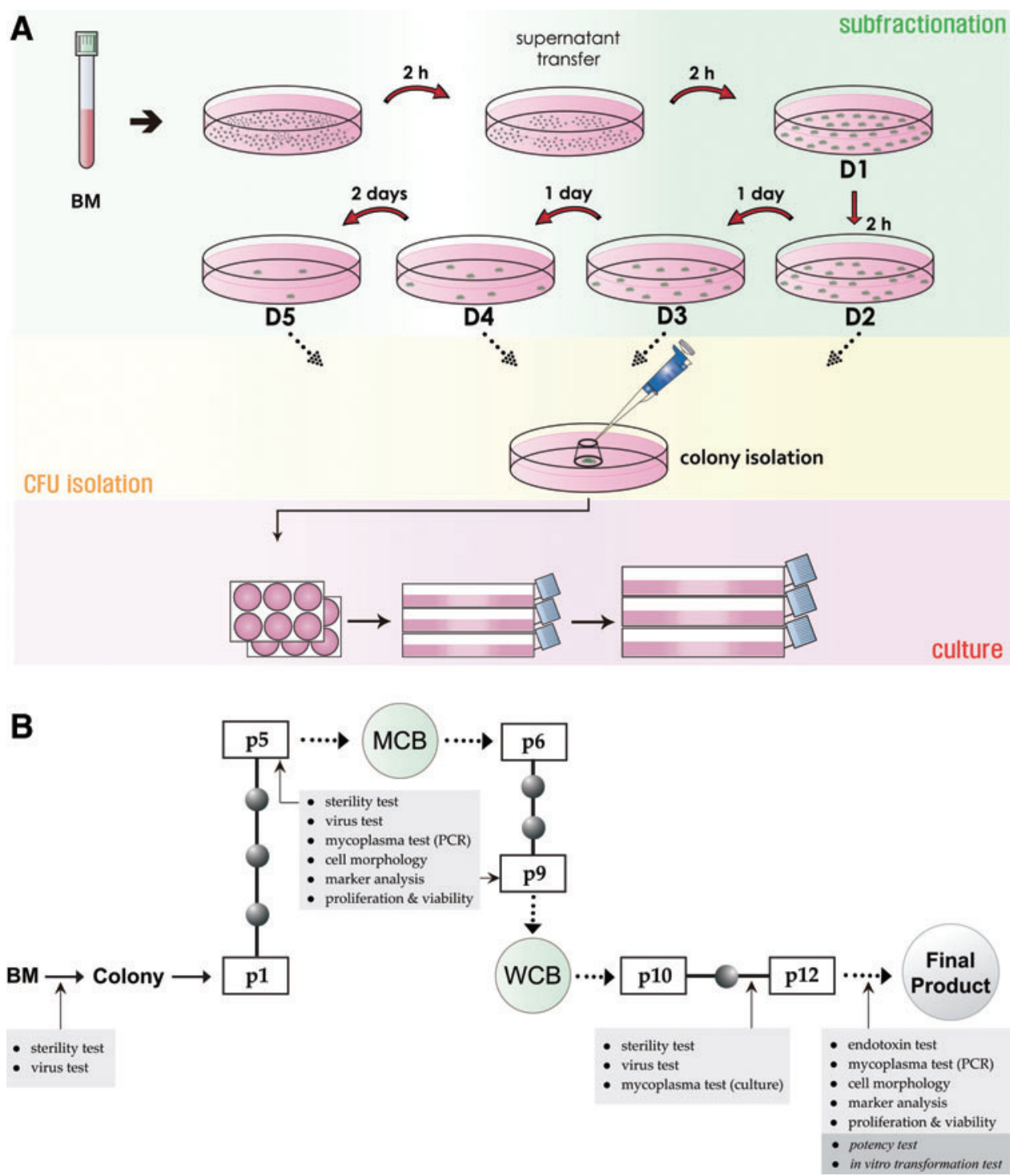

FIG. 1. Schematic representation of clinical-grade cMSC production and quality control processes in a good manufacturing practice facility. (A) cMSC production is composed of subfractionation, CFU isolation, and culture steps. Briefly, bone marrow aspirates was mixed with isolation medium followed by incubation in a 100-mm culture dish. At each time point indicated, only the supernatant, which contained floating nonadherent cells without the cells settled to the bottom, was transferred to new dishes. Incubation was carried out for 10-14 days until colonies appeared. Only well-separated single colonies with a diameter of about $4 \mathrm{~mm}$ in each dish were selected, detached, and isolated using cloning cylinders. The dissociated cells were transferred to six-well plates and then to larger culture flasks for culture expansion. When the cells reached 70-80\% confluence, they were trypsinized and replated for further expansion. (B) Two-point cell banking, including MCB and $\mathrm{WCB}$, is a key step in our production and quality control. MSCs harvested at passages 5 and 9 were cryopreserved for MCB and WCB, respectively. The final cMSC products were produced from cells obtained at passage 12 . To ensure high quality and safety of the final cell products for the patients, a stringent quality control system was strategized as indicated at each step (For full details, refer to the text). The final cMSC products should meet all of these criteria. BM; CFU, colony forming unit; cMSC, clonal mesenchymal stem cell; MCB, master cell bank; MSC, mesenchymal stem cell; WCB, working cell bank. Color images available online at www.liebertpub.com/tec

was set within 72 to $120 \mathrm{~h}$. Further cell expansion was done in a $175-\mathrm{cm}^{2}$ culture flask at a seeding density of $0.5-1 \times 10^{6}$ cells $/ 175-\mathrm{cm}^{2}$. Features of this GMP-compatible manufacturing process were two-point banking steps. Master cell bank (MCB) was the first cell freezing point for ensuring potential cell clones with good proliferation activity. Working cell bank (WCB) was planned for the second cell freezing point, from which the final cell products were to be produced.
Cells obtained at passages 5 and 9 were cryopreserved in liquid nitrogen for $\mathrm{MCB}$ and $\mathrm{WCB}$, respectively. The final products were produced from cells harvested at passage 12 . Because GMP should ensure the quality and safety of the final cell products for the patients, a strictly-regulated quality control process was established in addition to the manufacturing process (Fig. 1B). When screening the source material, the presence of pathological microbes and viruses 


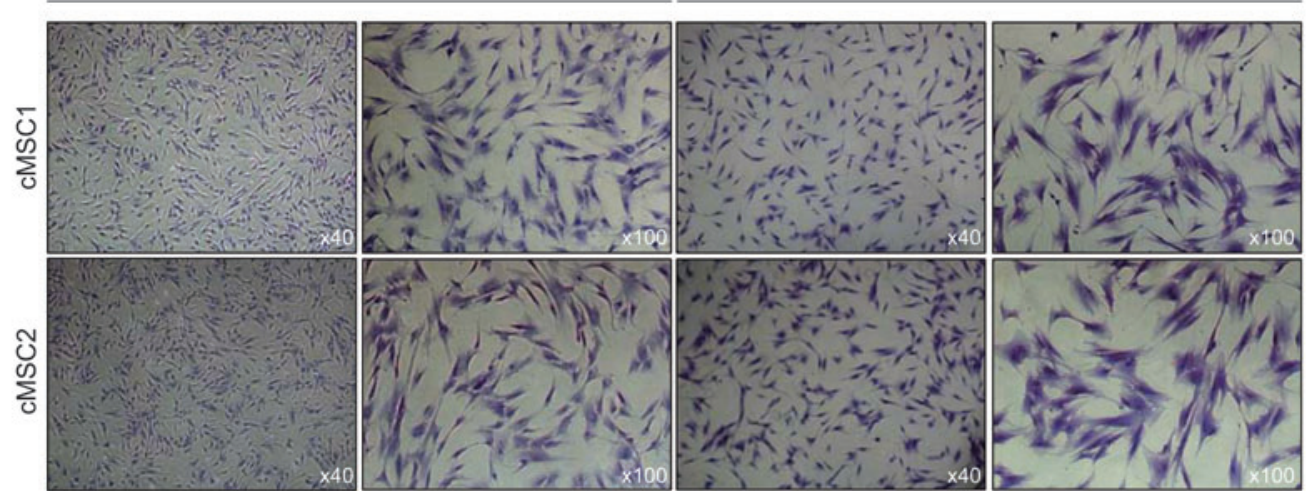

B cMSC1 cMSC2
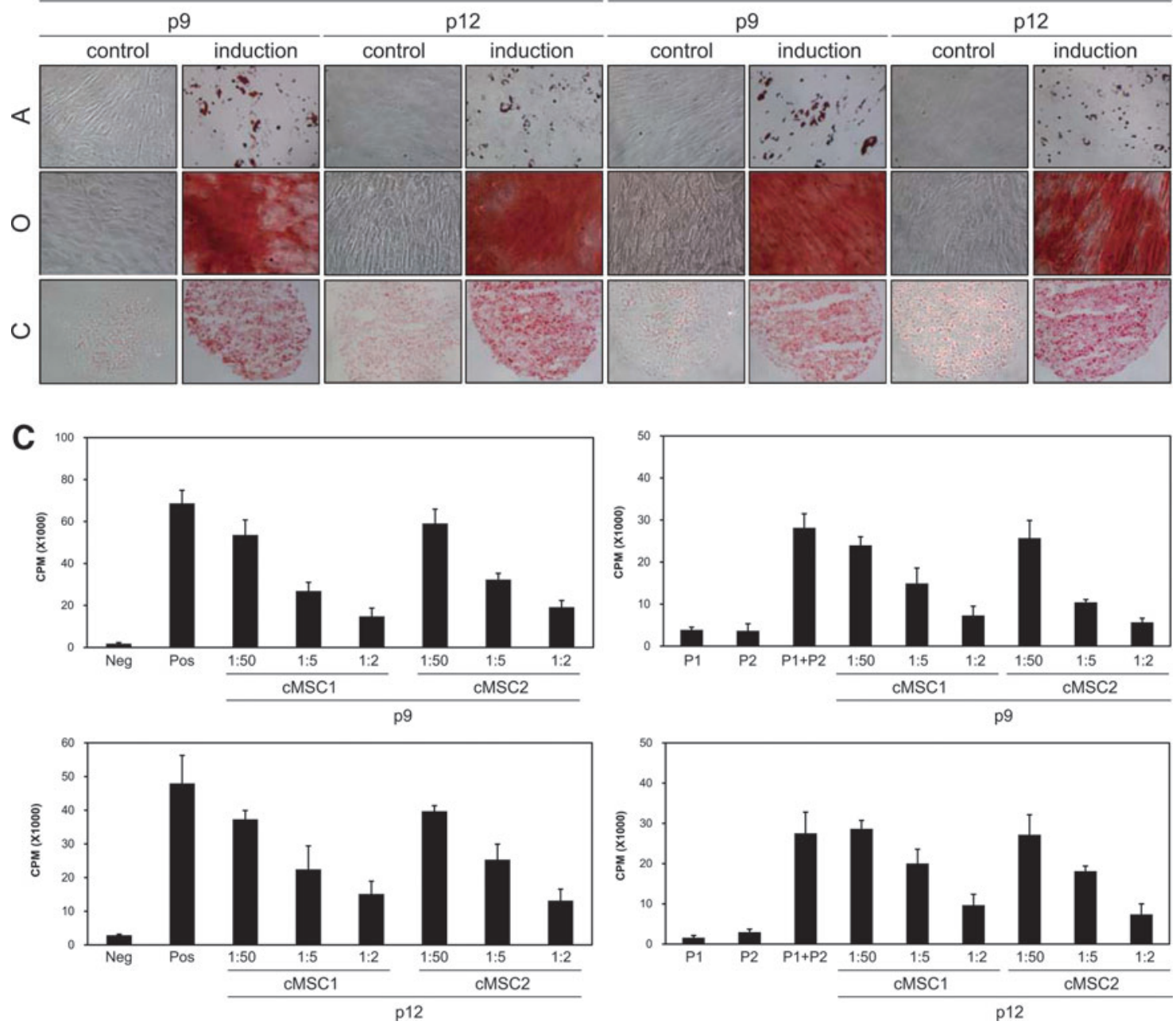

FIG. 2. Characterization and properties of clinical-grade cMSCs. (A) Two different MSC clones exhibited typical fibroblast-like appearance. The cells of each clone at passages 9 and 12 were stained with crystal violet for clear visualization under a light microscope. Magnification, 40× and 100×. (B) Differentiation potential of these cMSCs was evaluated by inducing them to differentiate into the three mesenchymal cell types: adipocytes $(\mathrm{A})$, osteoblasts $(\mathrm{O})$, and chondroblasts (C). Induced cells at both passages were determined by tissue-specific staining (adipocytes visualized using Oil Red O, osteoblasts by Alizarin Red S, and chondroblasts by Safranin O). Molecular marker expression for each differentiation was also examined (Supplementary Fig. S1). (C) In vitro immunosuppressive activity of cMSCs was measured by [ $\left.{ }^{3} \mathrm{H}\right]-$ thymidine incorporation. Peripheral blood mononuclear cells (PBMCs; $2 \times 10^{5}$ cells) were stimulated with phytohemagglutinin $(1 \mu \mathrm{g} / \mathrm{mL})$ (upper left panel, passage 9 cells; lower left panel, passage 12 cells) or a total of $2 \times 10^{5}$ PBMCs from two different donors $\left(1 \times 10^{5}\right.$ cells each) were mixed and cultured for a mixed lymphocyte reaction (upper right panel, passage 9 cells; lower right panel, passage 12 cells). cMSCs (from $4 \times 10^{3}$ to $1 \times 10^{5}$ cells) were cocultured at the ratio of $1: 2$ to $1: 50$ (cMSCs:PBMCs) in these reactions. $\left[{ }^{3} \mathrm{H}\right]$-thymidine $(1 \mu \mathrm{Ci} /$ reaction) was allowed to be incorporated for the last $12-$ $16 \mathrm{~h}$ of culture. Radioactivity was measured with a $\beta$-counter. Neg, negative control; Pos, positive control stimulated by phytohemagglutinin; P1, PBMCs from donor A; P2, PBMCs from donor B. 
Table 1. Analysis of Cell Surface Antigen Expression on Clinical-Grade Clonal Mesenchymal Stem Cells

\begin{tabular}{lrrrrr}
\hline & $c M S C 1(\%$ expressed $)$ & & \multicolumn{2}{c}{$c$ cMSC2 (\% expressed) } \\
\cline { 2 - 3 } \cline { 5 - 6 } Marker & \multicolumn{1}{c}{$p 9$} & $p 12$ & & $p 9$ & $p 12$ \\
\hline CD14 & 2.47 & 1.68 & & 2.64 & 2.01 \\
CD29 & 99.80 & 96.40 & & 99.70 & 93.50 \\
CD31 & 2.49 & 2.41 & & 1.94 & 2.11 \\
CD34 & 2.11 & 2.40 & & 2.05 & 2.83 \\
CD44 & 95.70 & 95.60 & & 94.50 & 95.40 \\
CD45 & 2.12 & 2.85 & & 2.43 & 2.56 \\
CD73 & 98.59 & 99.81 & & 99.91 & 99.59 \\
CD90 & 99.70 & 93.60 & & 98.50 & 97.00 \\
CD105 & 90.34 & 99.60 & & 99.41 & 93.49 \\
CD106 & 1.72 & 2.15 & & 2.46 & 2.49 \\
CD119 & 2.25 & 1.94 & & 3.12 & 2.34 \\
CD146 & 5.28 & 2.98 & & 7.43 & 3.50 \\
CD166 & 31.60 & 94.80 & & 25.30 & 84.30 \\
CD184 & 1.73 & 2.03 & & 2.56 & 1.93 \\
HLA-I & 18.50 & 32.60 & & 12.40 & 43.70 \\
HLA-DR & 1.92 & 1.59 & & 2.74 & 2.40 \\
\hline
\end{tabular}

cMSC, clonal mesenchymal stem cell.

was checked (Refer to Supplementary Materials and Methods for details). At passage 5 before the MCB storage, tests for pathologic microbes and mycoplasmas were performed, during which the fast assay for cytopathic effect (CPE) and hemadsorption were performed instead of the virus test. $\mathrm{CPE}$, which refers to the morphological change in the host cells caused by a viral infection, was assayed by culturing cMSC-treated human normal lung fibroblast cell line MRC5 and monkey kidney epithelial cell line, Vero, for up to 14 days to determine the infection. Measles virus and sendai virus were used as control viruses. The hemadsorption assay checked the ability of the host cells, infected by the hemagglutinin-producing virus, to adsorb erythrocytes. At the same time, the cultured cMSCs were checked for the homogeneous fibroblast-like cell morphology, MSC marker analysis for MSC identification and purity, and cell viability. The cells for MCB storage must meet all of the preset criteria. If the cells fail to meet any of preset specifications, the cells are discarded immediately. At passage 9 before WCB storage, the same tests as for MCB storage were performed. Normal virus test was added at this step. A third test was done between passages 11 and 12. As for the source material screening, sterility and virus tests were performed. During this process, mycoplasmas were additionally screened by the culture method. When the final cell products were released from passage 12, the existence of endotoxin and mycoplasma were checked. In parallel, the quality of the cultured cells was tested in terms of cell morphology, MSC marker analysis, and cell viability. In vitro transformation and potency tests were carried out for an MCB batch and a WCB batch, respectively. The cMSCs produced in our GMP facility met all of these criteria.

\section{Characterization of clinical-grade cMSC products}

Two clones named cMSC1 and cMSC2 were used for characterization. Our standards for MSC identification and characterization included four criteria: cell morphology, cell surface marker expression, differentiation potential, and immunosuppression. Plastic-adherent cMSCs exhibited fibroblastlike shapes. They retained fibroblastic morphology at passages 9 and 12 (Fig. 2A), indicating that the morphological consistency of cMSCs is maintained during all the passages of subculture. Second, the cell surface marker expression was analyzed using flow cytometry. Two clones were positive for CD29, CD44, CD73, CD90, CD105, CD146, CD166, and HLA-Class I, but were negative for CD14, CD31, CD34, CD45, CD106, CD119, CD184, and HLA-DR (Table 1). Each cell surface marker showed a constant expression at different passages $(9$ and 12) and between different clones, indicating a homogeneous population of cells during the production process.

Next, the differentiation potential of these cMSCs was examined. Although over-cultured MSCs reportedly show reduced differentiation ability, we harvested cells at late passages ( 9 and 12) to induce them to differentiate into the three mesenchymal cell types. The induced cells at both passages were determined by tissue-specific staining (adipocytes visualized using oil red $\mathrm{O}$, osteoblasts by alizarin red S, and chondroblasts by safranin O) (Fig. 2B). The results showed unchanged differentiation capability of each clone even at passage 12, which was further supported by molecular marker expression for each differentiation (Supplementary Fig. S1). Finally, the in vitro immunosuppressive activity was measured. The immunosuppression was estimated by suppression of lymphocytic proliferation caused by a mitogenic stimulation and by a mixed lymphocyte reaction. In both reactions, lymphocytic proliferation was significantly inhibited when they were cocultured with cMSCs in a cell number-dependent manner (Fig. 2C). Collectively, these results indicated that our manufacturing procedures produce the final cMSC products meeting all of our preset criteria of morphology, marker expression, differentiation, and immunosuppression.

\section{Safety evaluation of clinical-grade cMSC products}

One of the main objectives of GMP production is to provide safe products for patients. For the safety of the final products, we evaluated their toxicity in immunodeficient athymic nude mice (Table 2). The toxicity test was performed in a certified good laboratory practice facility. The tests comprised single injection toxicity, multiple injection toxicity, biodistribution and cell detection, and tumorigenicity in vivo. Chromosome analysis including karyotyping and oligo-fluorescence in situ hydridization (oligo-FISH) was independently performed. In all these tests, the test substance (TS) was prepared by suspending the final cMSC products in normal saline for injection and was intravenously injected into mice through the tail veins.

A single injection toxicity test was designed to evaluate the acute toxicity of TS. Three doses of cMSCs $\left(5 \times 10^{4}\right.$ cells $/ 300 \mu \mathrm{L}$ normal saline for injection/head for low-dose, $5 \times 10^{5}$ for medium-dose, and $1 \times 10^{6}$ for high-dose) were injected into the female and male mice, and then the mice were observed up to 14 days. During the observation period, there were no deaths in the control and stem cell-treated groups. Neither clinical abnormality nor significant weight change was evident. Necropsy of all animals at 14 days showed no gross findings, indicating no acute toxicity of the cMSC products (Table 2). 


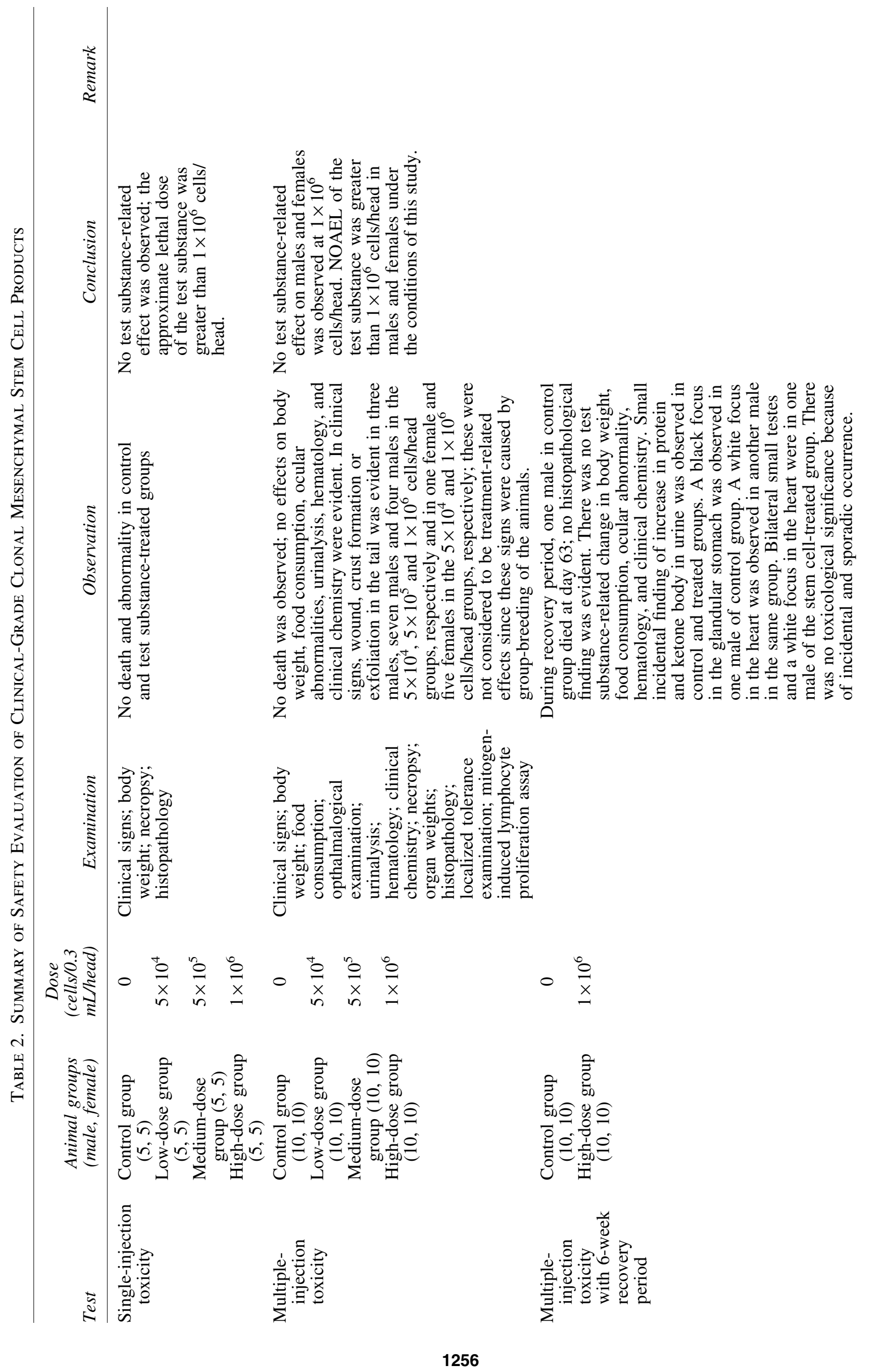




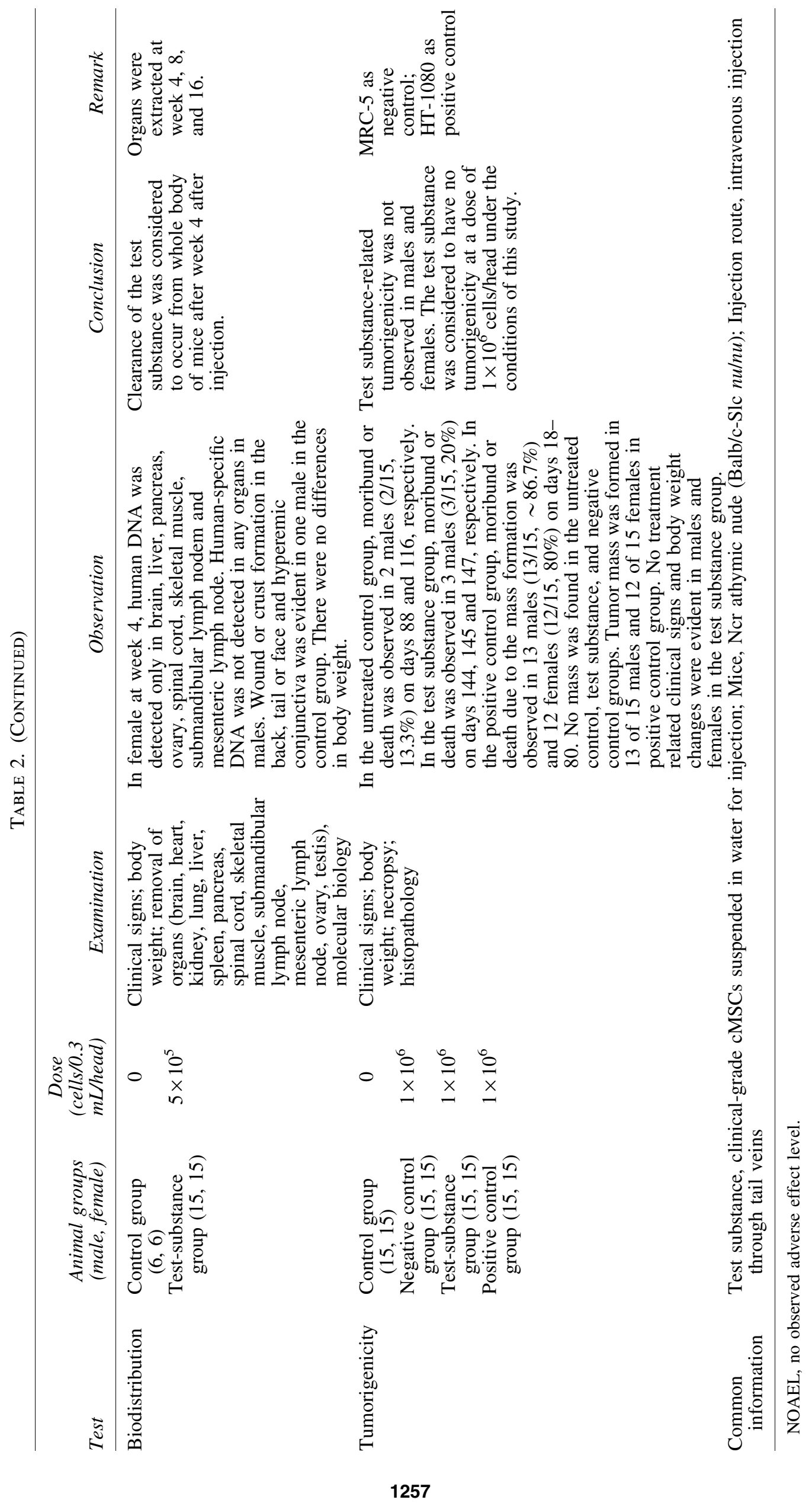


The multiple injection toxicity test was conducted with the low, medium, or high doses of TS (Table 2). Each dose of TS was injected three times at an interval of 2 weeks, based on the required time for production of large amounts of the final cMSC products from WCB cells. For acute toxicity evaluation, all mice were examined for 4 weeks for clinical signs including body weight, food consumption, ophthalmological examination, urinalysis, hematological examination, clinical chemistry, necropsy for gross pathology, organ weights, and histopathology. No death was observed. There were no effects on body weights, food consumption, and ocular abnormalities during the observation period. No change in urinalysis, hematology, and clinical chemistry data associated with the cMSC injection was observed throughout the course of the test. No cMSC-related effects on organ weights were evident in any animals. Furthermore, no gross finding or lesion in necropsy and histopathological examination was considered attributable to TS. In mitogen-induced lymphocyte proliferation assays, splenic $\mathrm{T}$ and $\mathrm{B}$ lymphocytes remained unchanged in males and females in the treated groups as compared with the control group. For long-term toxicity evaluation, we examined other sets of control and high-dose groups with a recovery period of 6 weeks later, following third administration of the high-dose TS. During the recovery period, one male in the control group died on day 63 and no histopathological finding could clearly indicate the cause of death. No abnormal clinical sign was found in TS-treated group. There was no cMSC-related change in body weight, food consumption, ocular abnormality, hematology, and clinical chemistry. In necropsy, a black focus in the glandular stomach was observed in one male of the control group, which was caused by mucosal necrosis according to the histopathological analysis. A white focus in the heart was observed in another male in the same group. Small testes and a white focus in the heart were in one male of the cMSC-treated group. According to the histopathological examination, the small testes were associated with atrophy of seminiferous tubules, while the white focus in the heart was epicardial mineralization. However, there was no toxicological significance because of the incidental and sporadic occurrence. Local irritancy at the injection sites was not evident in any animal in TS-treated group. In mitogen-induced lymphocyte proliferation assays, proliferation of splenic $\mathrm{T}$ and $\mathrm{B}$ lymphocytes was not altered in any animals of the recovery groups treated with TS compared with control group. Collectively, there was no cMSC-related effect on mice at the high dose. The results indicated that the observed adverse effect level (NOAEL) of the cMSC products is smaller than $1 \times 10^{6}$ cells/head in mice under the conditions of this test (Table 2).

To trace the in vivo distribution of intravenously injected cMSC products, a test of biodistribution and cell detection was conducted for 16 weeks (Table 2). TS of medium dose was injected once into 15 males and 15 females. The control groups, composed of six males and six females, received normal saline for injection. Animals were necropsied at week 4, 8, and 16 postinjection. The main organs including brain, lungs, heart, liver, kidneys, spleen, pancreas, spinal cord, skeletal muscles, submandibular lymph nodes, mesenteric lymph nodes, testes, and ovaries were collected from each mouse. By using the genomic DNAs extracted from each organ, polymerase chain reaction analysis was performed to detect the distribution of cMSCs. The existence of incorporated cMSCs was detected with a human-specific primer for Alu. Template genomic DNA quality extracted from the mouse organs was assessed by a mouse-specific oncogenic gene MOS. Human Alu was not detected in any organs in males. In females at week 4, human DNA was detected only in the brain, liver, pancreas, ovaries, spinal cord, skeletal muscles, submandibular lymph nodes, and mesenteric lymph nodes, but it was no longer detected in the same organ samples at week 8 and 16 (Supplementary Table $\mathrm{S} 1)$. Consequently, clearance of human cMSCs from the whole body of athymic nude mice was considered to occur after week 4 postinjection.

Tumorigenicity is considered to be one of the most important hurdles in the clinical application of stem cells. ${ }^{18,19}$ In vivo tumorigenicity test was performed for 26 weeks. The cMSCs was intravenously injected into the mice to reflect the clinical route of administration at a high dose of $1 \times 10^{6}$ cells/head. As negative control, human embryonic lung fibroblast MRC-5 cells that do not form mass were subcutaneously injected at $1 \times 10^{3}$ cells/head. Human fibrosarcoma HT-1080 cells, which form tumor mass, were subcutaneously injected at $1 \times 10^{3}$ cells/head. Each group consisted of 15 males and 15 females. The evaluation parameters included clinical signs for 26 weeks, such as body weight, gross mortem examinations, and histopathological analyses of selected tissues. No mass was found in the untreated control, cMSC, and negative control groups, whereas a tumor mass was formed in 13/15 males and 12/15 females in the positive control group. No treatment-related clinical signs and body weight changes were evident in males and females in the cMSC group. Based on the histopathologic findings, there was no indication of cMSC-related neoplastic or non-neoplatic lesion in both sexes. The results indicated that cMSCs do not induce tumors at a dose of $1 \times 10^{6}$ cells/ head (Table 2). No anchorage-independent growth of cMSC products in the in vitro transformation assay using a soft agar culture was consistent with the in vivo data.

In vitro chromosomal analysis further supported the safety of the cMSC products produced in our manufacturing process. Two clones of cMSC products at passages 7, 9, and 12 were independently subjected to classical karyotyping to evaluate the gross numerical and structural stability of their chromosomes. No chromosomal aberration was observed in any sample (Fig. 3A). Despite its usefulness, classical karyotyping with G-banding analysis has shortcomings; it depends only on dividing cells and cannot detect cryptic rearrangements or aberrations covering small areas. As previously reported, a more sophisticated test incorporating in situ hybridization (FISH) to verify chromosomal abnormalities could supplement the genetic stability evaluation of the cMSC products. ${ }^{20}$ The in situ karyotyping using oligoFISH probes for enumerating chromosomes was conducted in the interphase nuclei of cMSCs at the same passages of the G-banding analysis. No aneuploidy was detected by in situ karyotyping, providing more reliable evidence of the genetic stability of the clinical-grade cMSC products (Fig. 3B).

\section{Practical production of clinical-grade cMSCs}

We next sought to determine the amount of cMSC products that can be practically manufactured using our GMP-compatible methodology based on the SCM. Three 


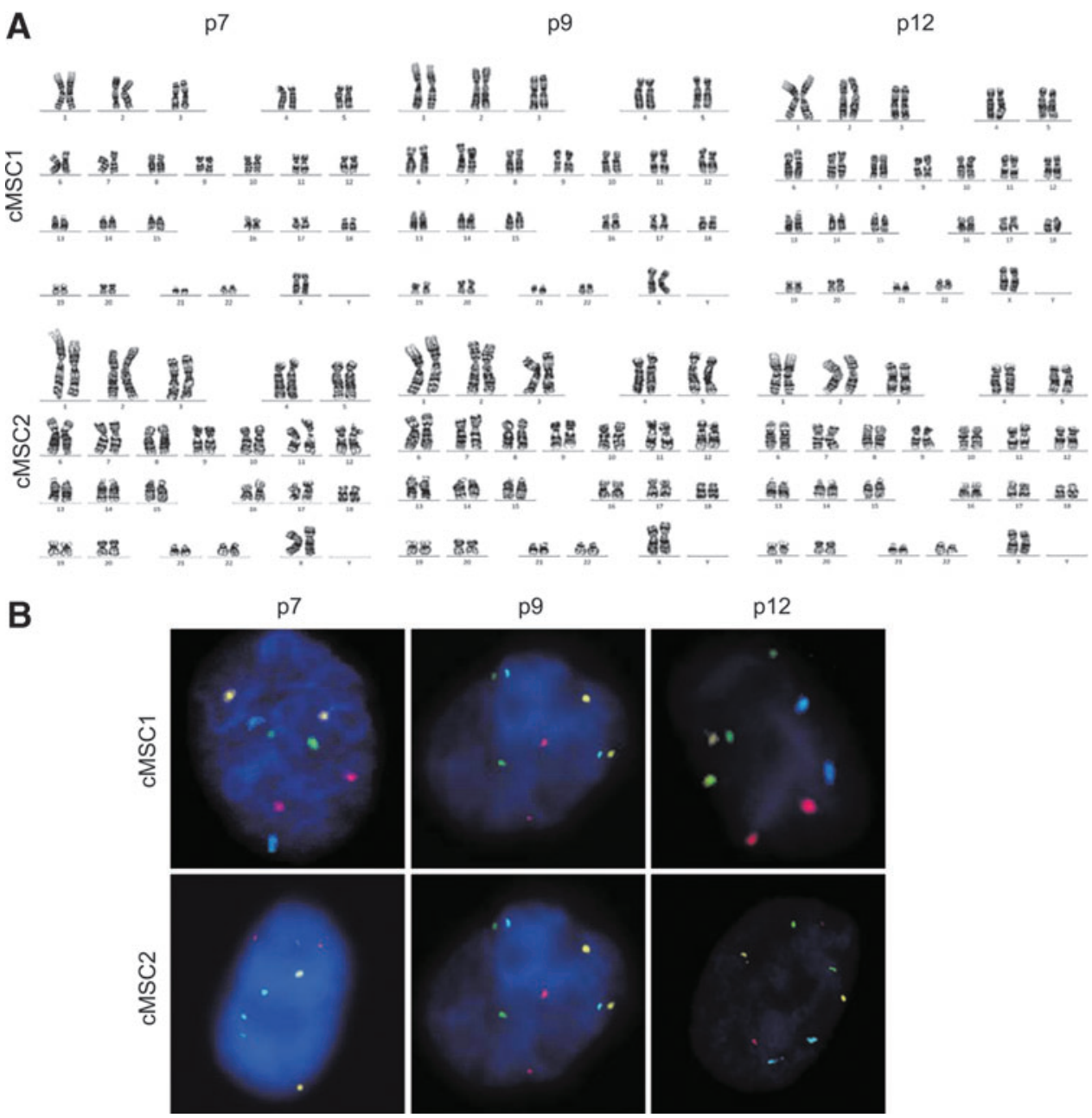

FIG. 3. Chromosomal analysis of clinical-grade cMSCs. (A) Classical karyotyping with G-banding was performed for two clones of cMSC products. The cells at three different passages $(7,9$, and 12) were independently subjected to karyotyping to evaluate the gross numerical and structural stability of their chromosomes. No chromosomal aberration was observed in any sample. (B) To supplement the G-banding analysis, another karyotyping assay was performed to verify chromosomal abnormalities. In situ karyotyping using oligo-FISH probes for enumerating chromosomes of interphase nuclei was conducted with cMSCs at the same passages of the G-banding analysis. Along with the G-banding results, no chromosomal abnormality was detected by in situ karyotyping. Normal diploid karyotype in chromosomes 6, 8, 9, and 11 was representatively shown. Aqua colors correspond to the centromeres of chromosome 6; gold colors to the centromeres of chromosome 8, green colors to q12 region of chromosome 9 (9q12), and red colors to the centromeres of chromosome 11. oligo-FISH, oligo-fluorescence in situ hydridization.

independent trials were conducted. As shown in Table 3, there was substantial variation in colonies that were isolated for further expansion among bone marrow donors. Among these colonies, $20-30 \%$ of the clones that fully met the MCB criteria were deemed to be suitable for MCB cryopreservation. Furthermore, only $25-45 \%$ of these MCBsuitable clones fulfilled the WCB requirements. The other clones that did not fulfill the requirements for the $\mathrm{MCB}$ or WCB were dropped out of the manufacturing process. However, the stock numbers of cell vials at the WCB were not proportional to the number of WCB clones, because the proliferation activity of each clone differed. For example, production batch-1 produced only 4 WCB clones, which in turn produced 1430 WCB cell vials. On the other hand, batch-2 produced 10 WCB clones, but only 719 cell vials were ultimately cryopreserved for the WCB due to the rel- atively lower proliferation activity of this batch compared with that of batch-1. This suggests that upon release of these cells to patients as the final products, a total of 474 product packages $\left(5 \times 10^{7}\right.$ cells per package per $50 \mathrm{~kg}$ human; the prospected clinical dose of cMSCs is $1 \times 10^{6}$ cells $/ \mathrm{kg}$ body weight of a patient) could be manufactured from three batches of cMSC production. Thus, about 470 patients (1 product per $50 \mathrm{~kg}$ patient) could be treated with this batch production. These data indicated that our GMP methodology is sufficient to produce cMSC products from small aspirates of the bone marrow for treating a large number of patients.

\section{Discussion}

In this study, we established a GMP-compatible manufacturing procedure for the clinical-grade production of human bone marrow-derived cMSCs based on the SCM 


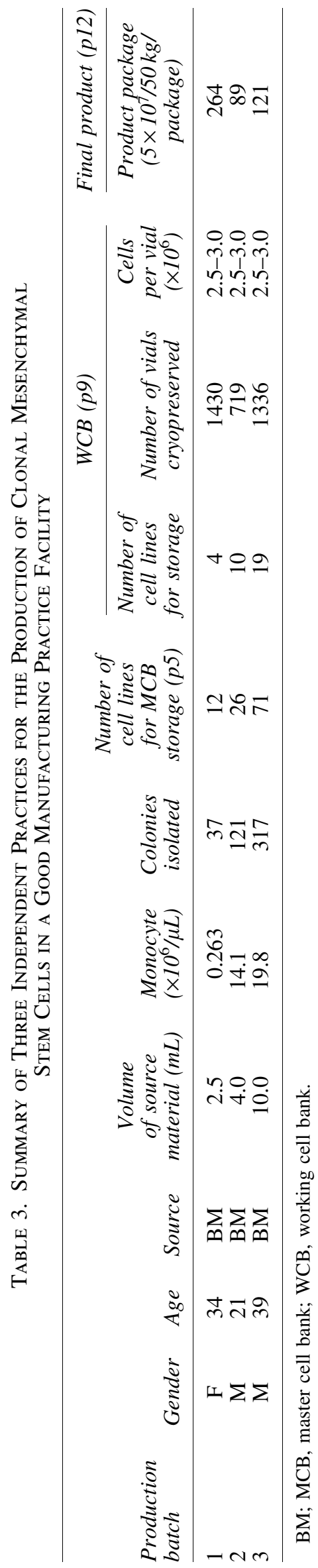

(Fig. 1). We optimized the culture procedures to expand and obtain a population of cMSCs from single CFU-derived colonies. The characterization results of the cMSCs in the MCB, WCB, and final products met our preset criteria of morphology, cell surface antigen expression, differentiation potential, and suppression of lymphocyte proliferation (Fig. 2 and Table 1). The results of the animal preclinical toxicity tests showed no toxicity and no tumor formation in vivo (Table 2). No chromosomal abnormalities were detected by in situ karyotyping (Fig. 3). The manufacturing and quality control data indicated that our GMP methodology can sufficiently produce cMSC products from a small amount of bone marrow aspirate to treat a number of patients (Table 3 ).

There are still several main hurdles to overcome in stem cell therapeutics with MSCs. These include the improvement of efficacy, setting up a standard manufacturing protocol, reducing the cost of large production, and elucidation of the treatment mechanisms. The clinical trials performed over the last decade have showed the safety of clinical-grade MSCs but mixed clinical outcomes have showed both optimistic and pessimistic prospects about the efficacy of MSC products. ${ }^{4-6,21,22}$ One of the possible reasons for mixed results may be the heterogeneity of the final MSC products. Due to the lack of MSC-specific isolation of the current isolation methods, the final MSC products produced by the conventional methods may contain heterogeneous populations of cells. A potential solution to overcome this hurdle may be to use cMSCs as the starting cell material to maximize the homogeneity of the final stem cell products. Bianco et al. recently pointed out that the MSCs prepared by conventional plating and culture methods cannot be assessed for their multipotency due to the heterogeneity of the cells, whereas only single colony-derived MSCs can be assessed for it. ${ }^{23}$ This argument suggests that if MSC therapeutics can be produced using single colony-derived MSCs, it may reduce the heterogeneity of MSC products. We previously developed an alternative culture protocol based on clonal selection to produce cMSCs from single CFU-derived colonies, different from the widely used conventional meth-

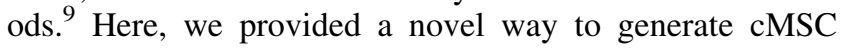
products for clinical purposes.

It is unfeasible to obtain a homogeneous population of MSC products with current isolation and culture technologies. Production of homogeneous MSC products is not theoretically possible even when individual clones of MSCs are used as the starting material, mainly because stem cells divide asymmetrically during the long mass culture process, and all differentiation- and senescence-related events are kinetically asymmetric. To date, no attempt has been made to produce MSC products with single CFU-derived colonies, probably because it is assumed that not enough cells can be produced to meet the demands of clinical trials. Here, we proved that a sufficient number of cMSC products can be manufactured with single CFU-derived colonies. This mass production of cMSCs using SCM was an unexpected result. A very large number of cMSCs can be produced by this standard manufacturing system in the absence of any special culture procedures or devices. Because a low percentage (5$10 \%$ ) of cell colonies showed such expansion capability, it is possible that the "true" MSCs have a much higher proliferation capacity than that of normal primary cells. In the future, we aim to compare the proliferative potential of 
cMSC lines in vitro. It is important to determine whether MSCs adapt to the in vitro culture environment and become part of a self-renewing pathway, thereby losing their nonself-renewing qualities.

Toxicological evaluation of stem cell products is very important step to determine the degree of toxicity and the relationship between the dose and adverse effects of stem cells administered. This evaluation also provides information on target organs and target functions, allowing for a scientifically supported extrapolation of the potential effects of these products in humans. In particular, the characterized risks need to be extrapolated to clinical situations and patients. The quality and reproducibility of safety data are key components of the utility of stem cell products to support the assumption of safety in humans. Our in vivo toxicity and tumorigenicity tests showed no severe toxicity and tumor formation even 6 months postinjection in mice. However, for these tests, we injected the final cMSC products at 2week intervals based on the time required to produce a large number of fresh cMSC products from the WCB. This experimental design could not test the cumulative dose effect on the cMSC products for injections administered over a shorter interval. Therefore, we are planning to test the toxicity and tumorigenicity of frozen cMSC products, which are designed for faster administration and are ready to be injected immediately, using a shorter interval. For the tumorigenicity test, we injected cMSCs intravenously to reproduce the administration route used in humans. However, we also need to test the tumor formation after multiple local injections to verify the "mass effect" of cMSCs, for accidental occurrences in clinical applications. Therefore, additional toxicity and tumorigenicity tests are required. In our protocol, the final cMSC products are produced at passage 12 . This raises a major concern about the possibility of transformation and chromosome abnormality of the cells. ${ }^{24,25}$ In situ karyotyping using oligo-FISH probes for chromosome enumeration indicated no aneuploidy of the cells at passages 7,9 , and 12 . We also further confirmed the chromosome stability of the cells at passage 14 and 15 (data not shown). In addition, the phase I clinical data with 10 GVHD patients who received the cMSC products confirmed their safety (in preparation).

As a technical note, the procedure applied here to generate cell colonies does not account for the density of cell plating at each supernatant transfer. It is possible that not all colonies were clonal populations originating from single cells. In particular, during the final passages of supernatant transfer, the floating cells could be dividing cells from the previous dish rather than small and/or less adherent cells. Therefore, an improved design would help set a precise density of cells at each supernatant transfer rather than just transferring the equally divided volume of supernatant; this would ensure a more systemic and effective selection of the clonal origin of each line.

The advantages of using a population of cMSCs for stem cell therapy and tissue regeneration are the following: First, it could reduce the possibility of causing immune reactions because other cell types are not contaminated. ${ }^{26}$ Second, it may increase the efficacy of MSC products, similar to more pure chemical drugs that have higher treatment efficacy. Third, patients may need a smaller number of MSCs to treat diseases because a population of cMSCs is used. Fourth, eliminating centrifugation, enzymatic treatment, and filtering procedures to obtain MSCs could lower the cost for large production. Fifth, a library of cMSC lines can be established and a specific MSC line having a greater potential to treat specific diseases or to induce tissue regeneration can be chosen. Overall, cMSC products could be a more efficacious and economic option in clinical settings.

Therapeutic stem cells are living drugs and are different from chemical drugs. The fundamental difference is that the components of the chemical drugs can be controlled whereas there is a limit to the regulation of stem cells. Due to this kind of inevitable limitation, it has been generous to developers of stem cell therapeutics to allow the use of heterogeneous stem cells. One solution for manufacturing highly "homogeneous" stem cell products is to use a population of clonal stem cells at the beginning of the manufacturing process. Additional efforts to manufacture more homogeneous stem cell products for their better safety and efficacy should be pursued. Here, we suggest a potential alternative to achieve this goal.

\section{Conclusions}

In this study, we established a GMP-compatible procedure for the clinical-grade production of human bone marrow-derived cMSCs based on the SCM. We optimized the culture procedures to expand and obtain a clonal population of final MSC products from single CFU-derived colonies in a GMP facility. The characterization results of the final cMSC products met our preset criteria. Animal toxicity tests showed no toxicity or tumor formation in vivo. No chromosomal abnormalities were detected, providing evidence of genetic stability of the clinical-grade cMSC products. The manufacture and quality control results indicated that our GMP methodology could produce sufficient clonal population of MSC products from a small amount of bone marrow aspirate to treat a number of patients.

\section{Acknowledgments}

This study was supported by the Bio \&Medical Technology Development Program (NRF-2011-0019634 \& NRF-2011-0019637) of the National Research Foundation by the Korean government (MEST), and by a grant from Inha University (44773-01).

\section{Disclosure Statement}

No competing financial interests exist.

\section{References}

1. Uccelli, A., Moretta, L., and Pistoia, V. Mesenchymal stem cells in health and disease. Nat Rev Immunol 8, 726, 2008.

2. Unger, C., Skottman, H., Blomberg, P., Dilber, M.S., and Hovatta, O. Good manufacturing practice and clinicalgrade human embryonic stem cell lines. Hum Mol Genet 17, R48, 2008.

3. Giancola, R., Bonfini, T., and Iacone, A. Cell therapy: cGMP facilities and manufacturing. Muscles Ligaments Tendons J 2, 243, 2012.

4. Ankrum, J., and Karp, J.M. Mesenchymal stem cell therapy: two steps forward, one step back. Trends Mol Med 16, 2032010. 
5. Allison, M. Genzyme backs Osiris, despite Prochymal flop. Nat Biotechnol 27, 966, 2009.

6. Rosenzweig, A. Cardiac cell therapy-mixed results from mixed cells. N Engl J Med 355, 1274, 2006.

7. Ra, J.C., Shin, I.S., Kim, S.H, Kang, S.K., Kang, B.C., Lee, H.Y., Kim, Y.J., Jo, J.Y., Yoon, E.J., Choi, H.J., and Kwon, E. Safety of intravenous infusion of human adipose tissuederived mesenchymal stem cells in animals and humans. Stem Cells Dev 20, 1297, 2011.

8. Wang, P., Li, Y., Huang, L., Yang, J., Yang, R., Deng, W., Liang, B., Dai, L., Meng, Q., Gao, L., Chen, X., Shen, J., Tang, Y., Zhang, X., Hou, J., Ye, J., Chen, K., Cai, Z., Wu, Y., and Shen, H. Effects and safety of allogenic mesenchymal stem cell intravenous infusion in active ankylosing spondylitis patients who failed NSAIDs: a 20-week clinical trial. Cell Transplant 23, 1293, 2014.

9. Song, S.U., Kim, C.S., Yoon, S.P., Kim, S.K., Lee, M.H., Kang, J.S., Choi, G.S., Moon, S.H., Choi, M.S., Cho, Y.K., and Son, B.K. Variations of clonal marrow stem cell lines established from human bone marrow in surface epitopes, differentiation potential, gene expression, and cytokine secretion. Stem Cells Dev 17, 451, 2008.

10. Nichols, J.E., Niles, J.A., DeWitt, D., Prough, D., Parsley, M., Vega, S., Cantu, A., Lee, E., and Cortiella, J. Neurogenic and neuro-protective potential of a novel subpopulation of peripheral blood-derived CD133+ ABCG2+CXCR4+ mesenchymal stem cells: development of autologous cell-based therapeutics for traumatic brain injury. Stem Cell Res Ther 4, 3, 2013 .

11. Anderson, P., Carrillo-Gálvez, A. B., García-Pérez, A., Cobo, M., and Martín, F. CD105 (endoglin)-negative murine mesenchymal stromal cells define a new multipotent subpopulation with distinct differentiation and immunomodulatory capacities. PLoS One 8, e76979, 2013.

12. Pittenger, M.F., Mackay, A.M., Beck, S.C., Jaiswal, R.K., Douglas, R., Mosca, J.D., Moorman, M.A., Simonetti, D.W., Craig, S., and Marshak, D.R. Multilineage potential of adult human mesenchymal stem cells. Science 284, 143, 1999.

13. Jung, K.H., Song, S.U., Yi, T., Jeon, M.S., Hong, S.W., Zheng, H.M., Lee, H.S., Cho, M.J., Lee, D.H., and Hong, S.S. Human bone marrow-derived clonal mesenchymal stem cells inhibit inflammation and reduce acute pancreatitis in rats. Gastroenterology 140, 998, 2011.

14. Kim, Y.M., Yi, T., Choi, J.S., Lee, S., Jang, Y.H., Kim, C.H., Song, S.U., and Lim, J.Y. Bone marrow-derived clonal mesenchymal stem cells as a source of cell therapy for promoting vocal fold wound healing. Ann Otol Rhinol Laryngol 122, 121, 2013.

15. Lim, J.Y., Yi, T., Choi, J.S., Jang, Y.H., Lee, S., Kim, H.J., Song, S.U., and Kim, Y.M. Intraglandular transplantation of bone marrow-derived clonal mesenchymal stem cells for amelioration of post-irradiation salivary gland damage. Oral Oncol 49, 136, 2013.

16. Yoo, H.S., Yi, T., Cho, Y.K., Kim, W.C., Song, S.U., and Jeon, M.S. Mesenchymal stem cell lines isolated by different isolation methods show variations in the regulation of graft-versus-host disease. Immune Netw 13, 133, 2013.
17. Jeon, M.S., Yi, T.G., Lim, H.J., Moon, S.H., Lee, M.H., Kang, J.S., Kim, C.S., Lee, D.H., and Song, S.U. Characterization of mouse clonal mesenchymal stem cell lines established by subfractionation culturing method. World $\mathbf{J}$ Stem Cells 3, 70, 2011.

18. Lee, A.S., Tang, C., Rao, M.S., Weissman, I.L., and Wu, J. C. Tumorigenicity as a clinical hurdle for pluripotent stem cell therapies. Nat Med 19, 998, 2013.

19. Ben-David, U., and Benvenisty, N. The tumorigenicity of human embryonic and induced pluripotent stem cells. Nat Rev Cancer 11, 268, 2011.

20. Hwang, S.M., See, C.J., Choi, J., Kim, S.Y., Choi, Q., Kim, J.A., Kwon, J., Park, S.N., Im, K., Oh, I.H., and Lee, D.S. The application of an in situ karyotyping technique for mesenchymal stromal cells: a validation and comparison study with classical G-banding. Exp Mol Med 45, e68, 2013.

21. Lalu, M.M., McIntyre, L., Pugliese, C., Fergusso, D., Winston, B.W., Marshall, J.C., Granton, J., and Stewart, D.J.; Canadian Critical Care Trials Group. Safety of cell therapy with mesenchymal stromal cells (SafeCell): a systematic review and meta-analysis of clinical trials. PLoS One 7, e47559, 2012.

22. Bernardo, M.E., and Fibbe, W.E. Safety and efficacy of mesenchymal stromal cell therapy in autoimmune disorders. Ann N Y Acad Sci 1266, 107, 2012.

23. Bianco, P., Cao, X., Frenette, P.S., Mao, J.J., Robey, P.G., Simmons, P.J., and Wang, C.Y. The meaning, the sense and the significance: translating the science of mesenchymal stem cells into medicine. Nat Med 19, 35, 2013.

24. Mishra, P.J., Mishra, P.J., Glod, J.W., and Banerjee, D. Mesenchymal stem cells: flip side of the coin. Cancer Res 69, 1255, 2009.

25. Tarte, K., Gaillard, J., Lataillade, J.J., Fouillard, L., Becker, M., Mossafa, H., Tchirkov, A., Rouard, H., Henry, C., Splingard, M., Dulong, J., Monnier, D., Gourmelon, P., Gorin, N.C., and Sensebé, L; Société Française de Greffe de Moelle et Thérapie Cellulaire. Clinical-grade production of human mesenchymal stromal cells: occurrence of aneuploidy without transformation. Blood 115, 1549, 2010.

26. Prockop, D.J., and Oh, J.Y. Mesenchymal stem/stromal cells (MSCs): role as guardians of inflammation. Mol Ther 20, 14, 2012.

Address correspondence to: Sun U. Song, PhD Translational Research Center Inha University School of Medicine 366 Seohaedaero Jung-Gu Incheon 400-712 Republic of Korea

E-mail: sunuksong@inha.ac.kr

Received: January 14, 2015

Accepted: September 2, 2015 Online Publication Date: November 5, 2015 